Report

\title{
Analysis of Generator Stator Remaining Breakdown Voltage for Power Plant
}

\author{
Kyeong-Yeol Kim, Tae-Sik Kong, Hee-Dong Kim, Jae-Heon Park, Tae-Sung Park
}

Korea Electric Power Corporation (KEPCO) Research Institute, Dae-jeon, South Korea

\section{Email address:}

kyeongyeol@kepco.co.kr (Kyeong-Yeol K.), kongts@kepco.co.kr (Tae-Sik K.), hdkim@kepco.co.kr (Hee-Dong K.), jh.p@kepco.co.kr (Jae-Heon P.), parkts@kepco.co.kr (Tae-Sung P.)

\section{To cite this article:}

Kyeong-Yeol Kim, Tae-Sik Kong, Hee-Dong Kim, Jae-Heon Park, Tae-Sung Park. Analysis of Generator Stator Remaining Breakdown Voltage for Power Plant. American Journal of Electrical Power and Energy Systems. Vol. 5, No. 3, 2016, pp. $22-27$.

doi: $10.11648 /$ j.epes.20160503.11

Received: July 3, 2016; Accepted: August 1, 2016; Published: August 31, 2016

\begin{abstract}
The reliability of stator windings is very important for stable operation of generators, while they are under thermal, mechanical, environmental, and electrical stresses over years of operation. Utility companies are very interested in the remaining breakdown voltage of generators and utilize the corresponding data for maintenance or replacement of generators. In most cases the remaining breakdown voltage is measured by performing a non-destructive dielectric strength test. In this paper, the remaining breakdown voltage of a generator is predicted using statistical methods by comparison with actual measured data, considering the cooling method, maker, and years of use of generators, as a means to supplement the existing methods. This method can be used to minimize the errors that may occur when only the dielectric strength test is used.
\end{abstract}

Keywords: Generator, Life Expectancy, Statistical Estimation, Stator Winding, Remaining Breakdown Voltage, Insulation Test, AC Current, Partial Discharge

\section{Introduction}

Accurate prediction of the remaining life of generator stator windings is a critical issue to the IPP service providers in the energy trade market in terms of asset management, because the dielectric breakdown failure of generators may result in enormous financial loss and huge impact on the energy supply plan. Proper maintenance and replacement of generator stator windings based on accurate prediction of remaining life is one of the key elements to maximize a return on investment. Insulation materials of stator windings go through the aging process affected by complex factors and the aging condition should be examined before the dielectric breakdown of the insulation materials occurs. Korean industry has used similar methods for the insulation test as the Japanese industry, where partial discharge $(\mathrm{Qm})$, tan delta $(\tan \delta)$, and $\mathrm{AC}$ current increase rate $(\Delta \mathrm{I})$ are used as the metrics for diagnosis. In this paper, dielectric degradation tests were conducted on a total of 354 generator samples. The results of these diagnosis tests were used for discharge-map lifespan analysis and several factors to consider in operation, design, manufacturing, and maintenance of generators are presented in this paper, taking into account the characteristics of years of operation, maker, and cooling method of each generator.

\section{Experimental Procedure}

Equipment used for non-destructive tests of generators are as follows. The polarization index (PI) was measured using a commercially available automatic insulation tester (Megger, $\mathrm{S} 1-5010)$ at DC $5 \mathrm{kV}$ in individual phases before applying AC voltage to the stator windings. Commercially available equipment, namely, Schering bridge (Tettex Instruments), coupling capacitor, and PD detector (Tettex Instruments, TE 571), were used to measure AC current, dissipation factor, and PD magnitude, respectively. The Schering bridge consists of a high voltage (HV) supply (Type 5283), a bridge (Type 2818), and a resonating inductor (Type 5285). A HV supply and control system (Tettex Instruments, Type 5284), Schering bridge (Tettex Instruments, Type 2816), resonating inductor 
(Tettex Instruments, Type 5288), coupling capacitor, coupling unit, and PD detector were used to measure AC current, dissipation factor, and PD magnitude in $15 \mathrm{kV}$ generator stator windings. The HV supply and control system, Schering bridge, and resonating inductor were used to obtain the $\mathrm{AC}$ current and dissipation factor measurements. For PD measurements, AC voltage was applied to the generator stator winding through a connected HV supply and control system. The coupling capacitor (Tettex Instruments, 4,000 pF) amplified signals from the winding, which were sent to the coupling unit (Tettex Instruments, AKV 572) and then to the PD detector (Tettex Instruments, TE 571) that measures the magnitude and pattern of PD. The frequency band of the PD detector ranged from 40 to $400 \mathrm{kHz}$. [1, 9, 10]

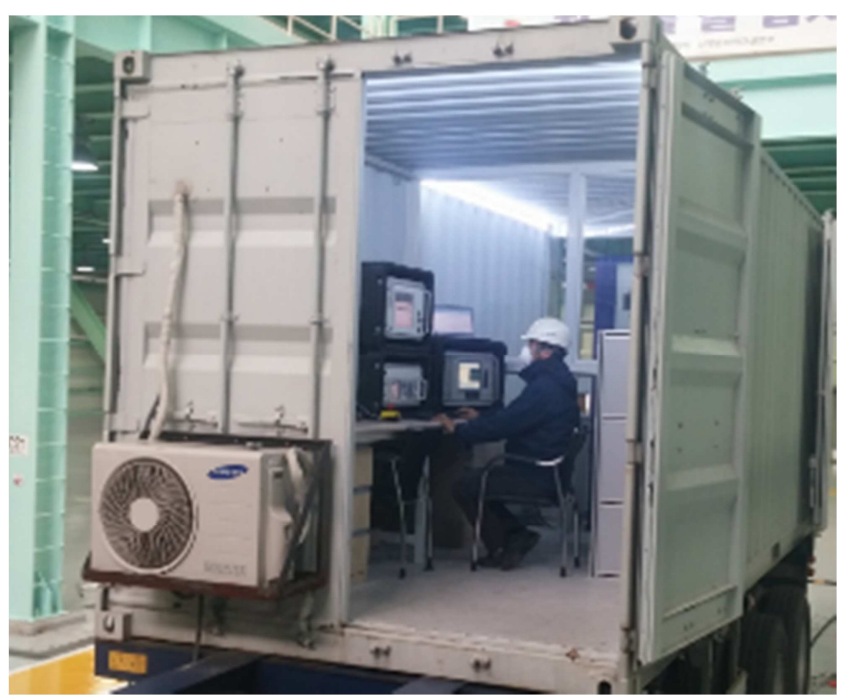

Fig. 1. The equipments of nondestructive test in $30 \mathrm{kV}$ generator stator windings.

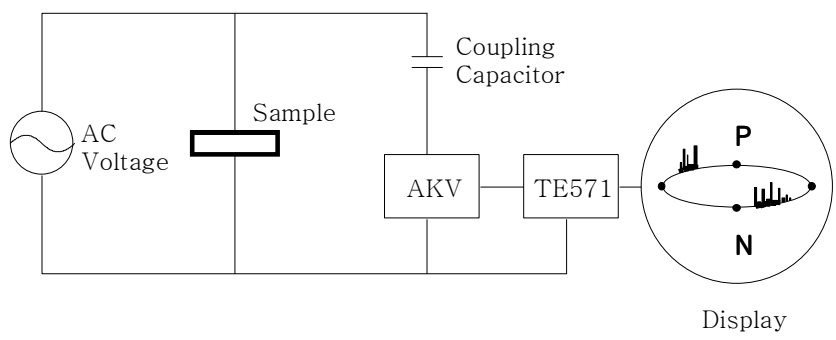

Fig. 2. Test circuit of partial discharge.

To check the insulation condition of the stator windings except for the bar with dielectric breakdown, the insulation resistance measurement, polarization index measurement, AC current increasing ratio test, dissipation factor tip-up measurement, and partial discharge measurement were performed. The AC current increasing ratio test consists of measurement of the increment in the current as the test voltage is increased, as shown in Figure 3. The dissipation factor tip-up test consists of measurement of the increment in the dissipation factor $(\tan \delta$ ) with respect to its value at the initial test voltage, as shown in Figure 4. The AC current increasing ratio $(\Delta \mathrm{I})$ and dissipation increment factor $(\Delta \tan \delta)$ are closely related to the partial discharge. There is no partial discharge at low voltage, but partial discharge begins to occur at the void within the insulation system as the test voltage is increased, eventually increasing the values of the AC current and dissipation factor. Because these test measure the current and dissipation factor of the whole insulation system, it is used to find the average deterioration condition of the insulation material. $[1,9,10]$

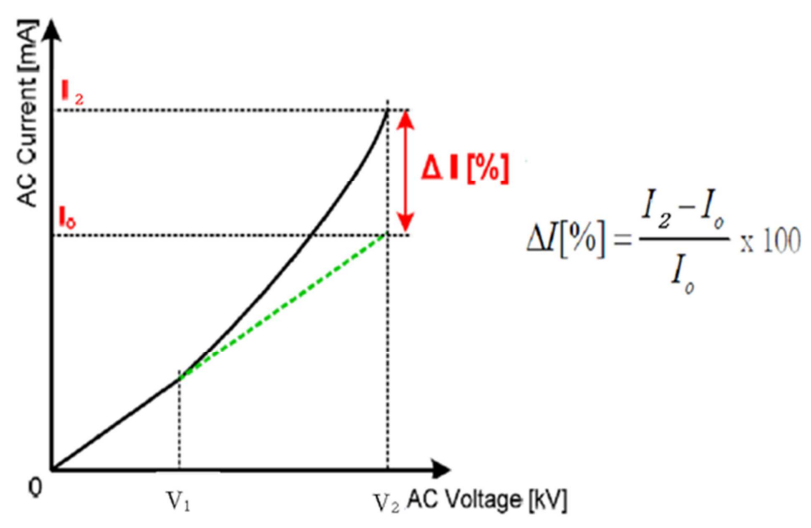

Fig. 3. Voltage vs. AC current.

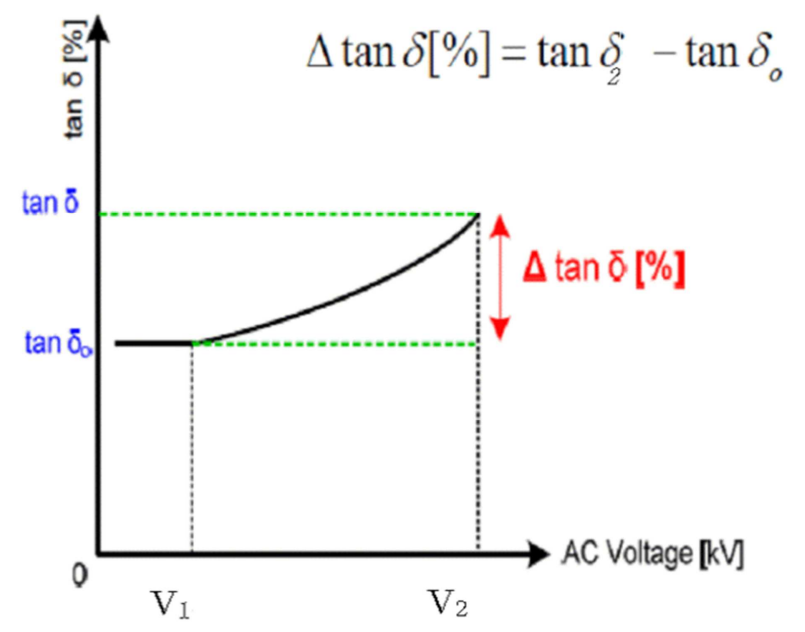

Fig. 4. Voltage vs. Dissipation factor.

Table 1. Insulation Test Method and Test Voltage.

\begin{tabular}{ll}
\hline Item & Test Voltage \\
\hline Polarization Index & $\mathrm{DC} 5 \mathrm{kV}$ \\
AC Current $\Delta \mathrm{I}[\%]$ & $\mathrm{E} / \sqrt{3} \times 1.25$ \\
Dissipation Factor $\Delta \tan \delta[\%]$ & $\mathrm{E} / \sqrt{3} \times 1.25$ \\
Maximum Partial Discharge $[\mathrm{pC}]$ & $\mathrm{E} / \sqrt{3}$ \\
\hline
\end{tabular}

\section{Test Results and Discussion}

\subsection{Analysis Method}

The discharge-map method as shown in Fig. 5 was used in order to calculate the remaining breakdown voltage of the generator in this paper. The discharge-map method uses the phenomena that the amount of void between insulation layers increases due to the aging of insulation materials, and dielectric loss increases over the applied voltage or capacitive current of dielectric materials increases as a result of void discharging. Assuming that the local voids diffuse inside the 
insulation materials along with the progression of aging process, the level of maximum partial discharge will increase, then the remaining breakdown voltage can be evaluated using Qmax, which is the void that makes the highest level of discharge, and $\Delta$, which is the coefficient that is the sum of $\Delta \tan \delta$ and $\Delta \mathrm{I}$.

$$
V_{R}=100-1.8(\Delta-0.8)-27.4 \log \left(\frac{\mathrm{Qm}}{1500}\right)
$$

$V_{R}$ : Remaining breakdown voltage (\%)

Qm: Electric Charge of Maximum Discharge (pC)

$\Delta$ : Discharge Parameter $(\Delta \tan \delta+\Delta \mathrm{I})(\%)$

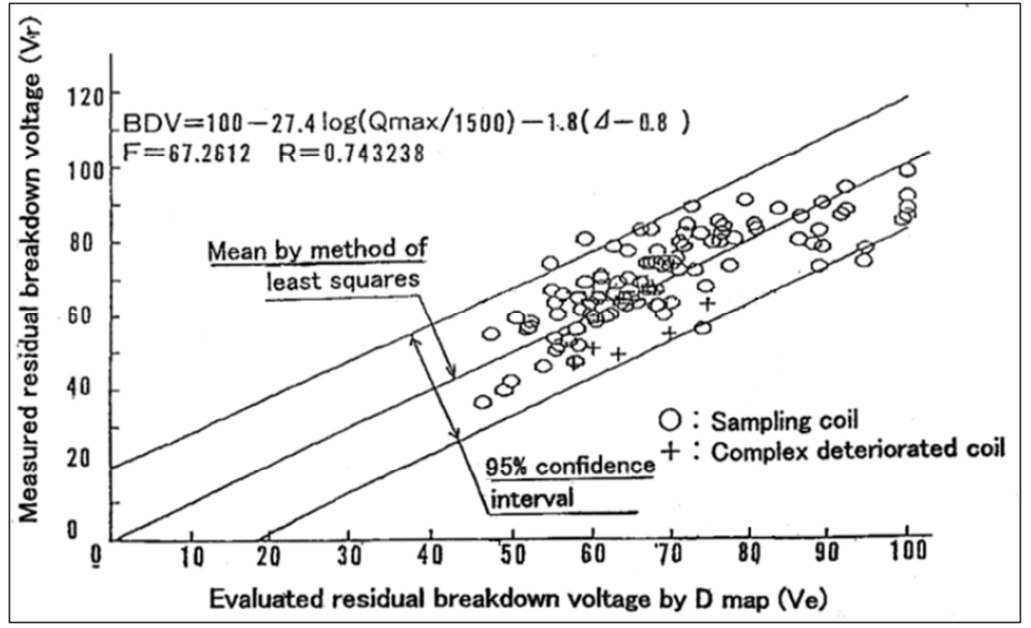

Fig. 5. Measured Remaining Breakdown Voltage.

Total number of generator samples for this analysis was 354 , including four makers of water cooling type, three makers of hydrogen cooling type, and two makers of air cooling type. Years of operation of generator of water cooling type was 0.3 $\sim 31$, that of hydrogen cooling type was $0.6 \sim 42$, that of air cooling type was $0.8 \sim 19$ years in this analysis.

Table 2. Generator Insulation Test Samples.

\begin{tabular}{lll}
\hline Cooling Type & Maker & Count \\
\hline Water Cooling & 4 & 139 \\
Hydrogen Cooling & 3 & 128 \\
Air Cooling & 2 & 87 \\
\hline
\end{tabular}

\subsection{Analysis Results}

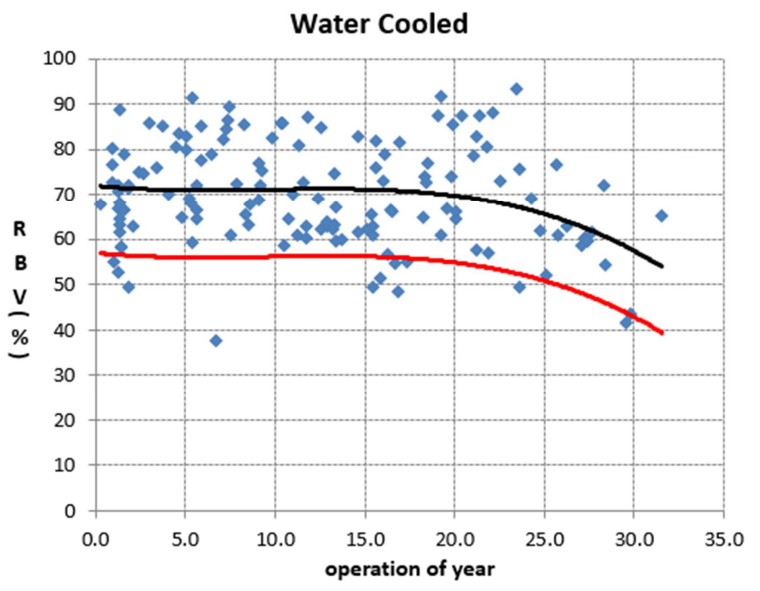

Fig. 6. Water-Cooled Generator RBV.

Four of the generator makers providing water-cooled generators to the Korean market were selected in this analysis, and the estimated remaining breakdown voltage of water-cooled generators are shown in Fig. 6. Overall the analysis shows that remaining breakdown voltage is over $60 \%$ after 30 years of average lifespan of generators. It was confirmed that the remaining breakdown voltage of generators mainly depends on the manufacturing quality of each maker and also the lifespan of water-cooled generators did not decrease sharply over time, rather it decreased gradually.

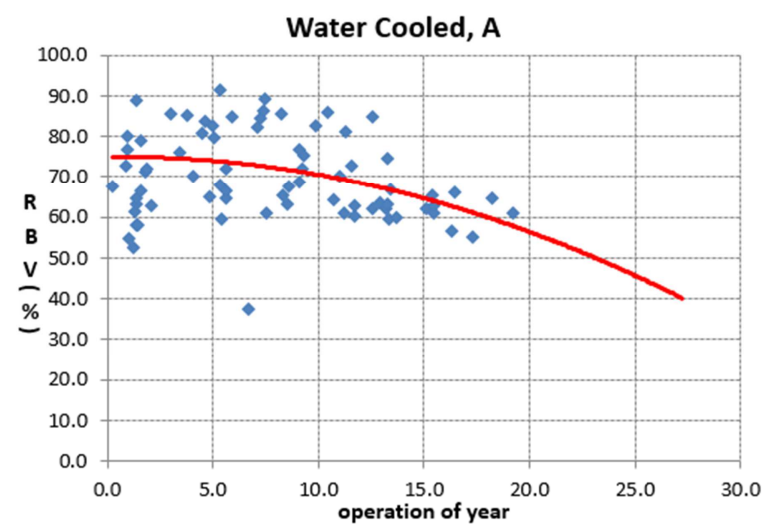

Fig. 7. Water Cooled Generator RBV (Maker A).

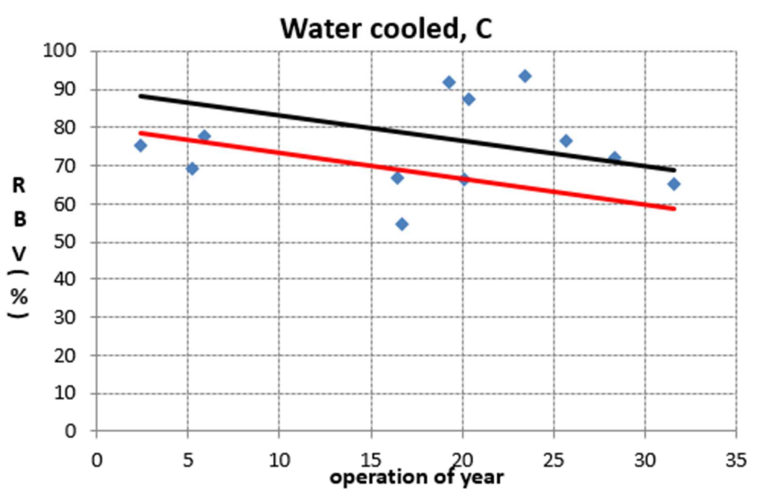

Fig. 8a. Water-Cooled Generator RBV (Maker C). 
The results also showed that the remaining breakdown voltage of water-cooled stator windings manufactured by a certain maker was reduced to $60 \%$ on average in $15 \sim 20$ years unlike other makers as shown in Fig. 7. This is consistent with the report that dielectric breakdown is frequent for water-cooled generators aged 15 years or older. As shown in Fig. 8, the remaining breakdown voltage of water-cooled generators manufactured by maker $\mathrm{C}$ is relatively high at the end of 30 years of life span, and problems caused by leaked cooling water in stator windings rarely occurs for certain makers.

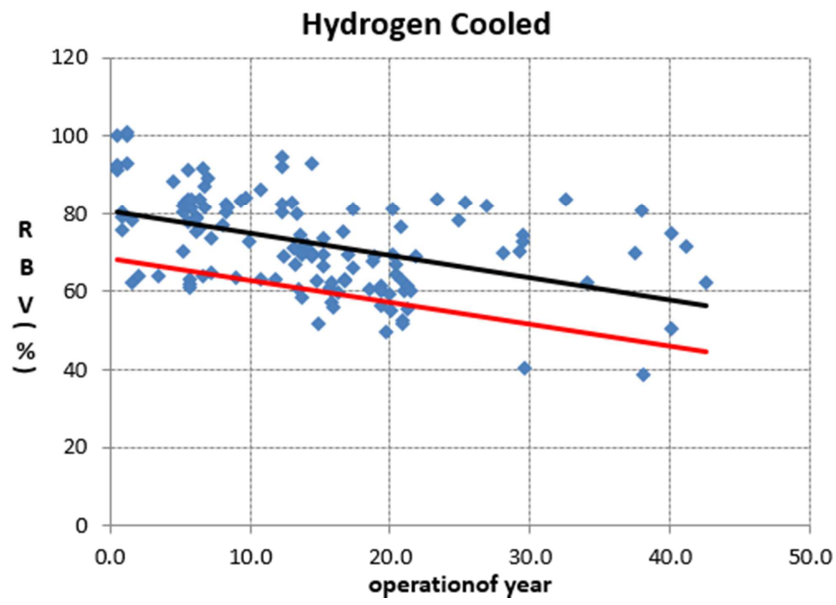

Fig. 8b. Hydrogen-Cooled Generator RBV.

Figure 8 shows the remaining breakdown voltage of generators of three hydrogen-cooled generator makers. The remaining breakdown voltage of hydrogen-cooled generators after 30 years is mostly higher than $60 \%$ because the penetration of foreign matter into the generators is prevented and there is no problem caused by the leaked cooling water. Unlike common predictions that the life span of water cooled generators would be shorter because of leaked cooling water, the average remaining breakdown voltage of water-cooled and air-cooled generators were similar in this analysis, while that of the water-cooled generators was higher than air-cooled generators by about $10 \%$.

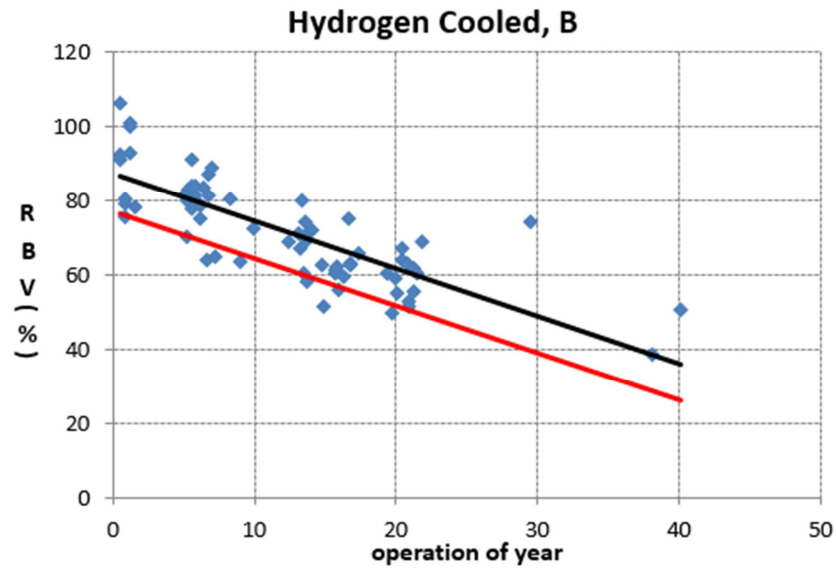

Fig. 9. Hydrogen-Cooled Generator RBV (maker B).

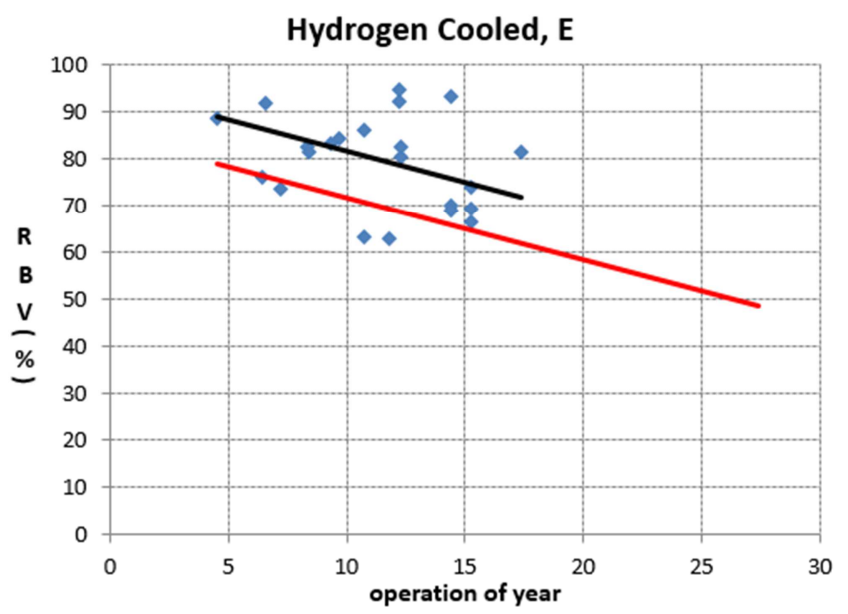

Fig. 10. Hydrogen-Cooled Generator RBV (maker E).

As shown in Figs. 9 and 10, it was confirmed that remaining breakdown voltage of hydrogen-cooled generators depended on the manufacturing process or design of each maker.

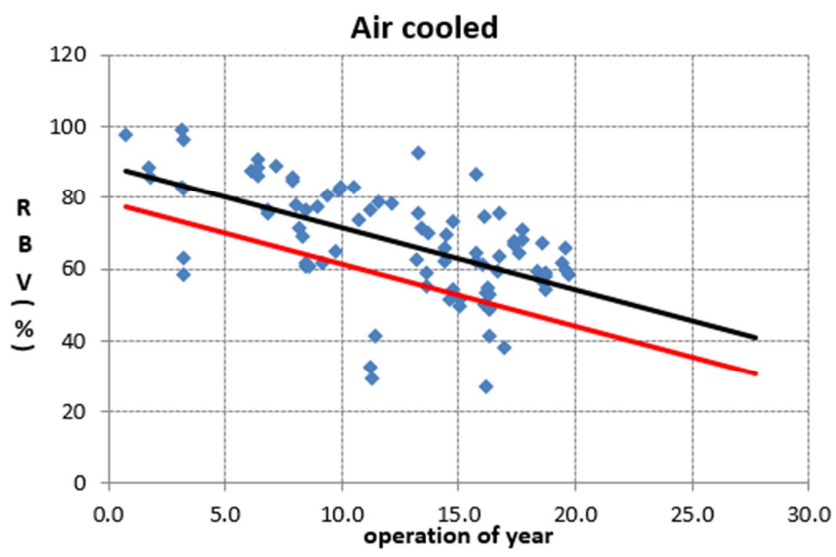

Fig. 11a. Air-Cooled Generator RBV.

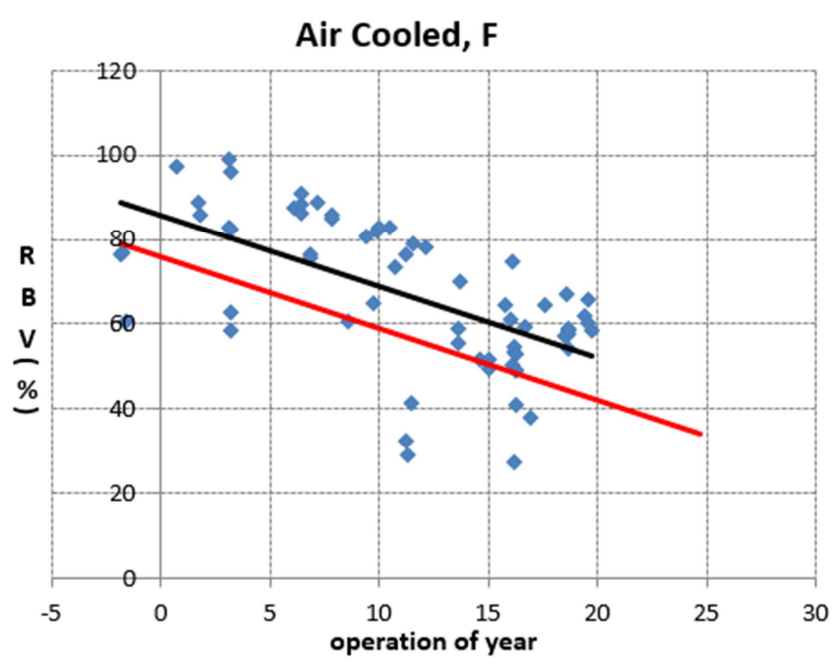

Fig. 11b. Air-Cooled Generator RBV (maker F).

Fig. 11 shows the characteristics of air-cooled generators manufactured by two makers. Traditionally generator makers have provided air-cooled generators to reduce the cost of hydrogen-cooled generators, but as shown in this paper 
air-cooled generators are greatly affected by the installation environment including pollution in windings since the stator windings are exposed to external air that is supplied through filters. In addition vacuum pressure impregnation method that is used for further cost reduction accelerates partial discharge and the reduction of remaining breakdown voltage over the years is relatively higher than other cooling types.

As shown in Figs. 11 and 12, the characteristics of air-cooled generators of different makers were similar without significant differences, which suggests that it depends more on the environment than manufacturing quality.

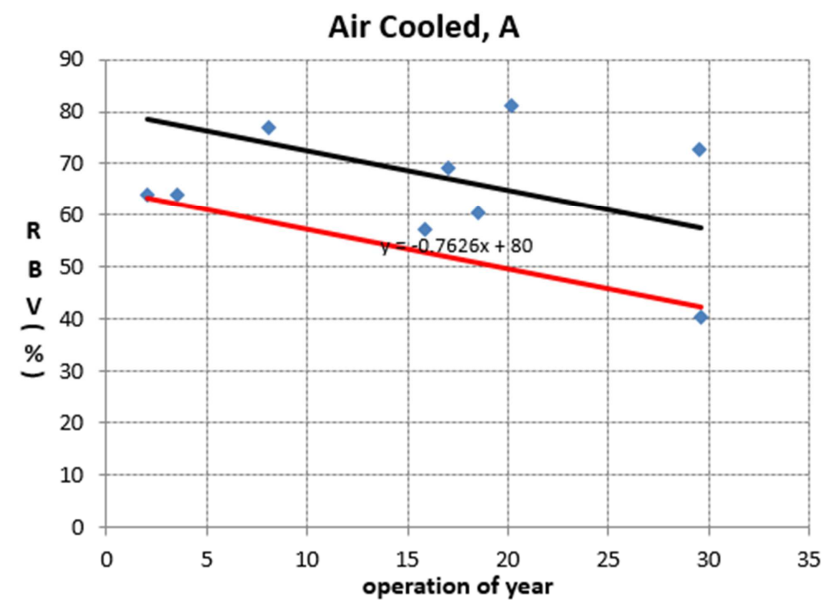

Fig. 12. Air-Cooled Generator RBV (maker F).

\section{Conclusion}

This paper presented a statistical asset management method that improves accuracy through comparison between remaining breakdown voltage data measured by users and that of similar generators, which improves the conventional asset management method using insulation tests or the discharge-map method. Furthermore the differences in remaining breakdown voltage between different stator windings cooling types were examined and they can be used as basic data for utility companies to make the proper selection in terms of lifespan and cost. Hydrogen cooling might be the best choice when small-scale generators are necessary, and specific winding method that minimizes the leakage of cooling water around the waterclip area should be selected for large-scale water-cooled generators. Dielectric breakdown test data taken from the generators close to the end of their life cycle are required to secure more accurate remaining breakdown voltage data.

\section{References}

[1] Tae-Sik Kong, Hee-Dong Kim, Tae-Sung Park, Kyeong-Yeol Kim, Ho-Yol Kim, "Analysis of Partial Discharge Patterns for Generator Stator Windings", American Journal of Electrical Power and Energy Systems Volume 4, Issue 2, March 2015, Pages: 17-22.

[2] G. C. Stone, E. A. Boulter, I. Culbert and H. Dhirani, Electrical
Insulation for Rotating Machines: Design, Evaluation, Aging, Testing, and Repair, Wiley-IEEE Press, 2003.

[3] G. C. Stone and I. Culbert, "Prediction of stator winding remaining life from diagnosis measurement," IEEE Int'l. Sympos. Electr. Insul. Conf., pp. 1-4, 2010.

[4] G. C. Stone 1993, "The Statistics of Aging Models and Practical Reality," IEEE Transactions on Electrical Insulation, vol. 28, no. 5, pp. 716-728, October 1993.

[5] C. Sumerer, "Statistical Lifetime of Hydro Generators and Failure Analysis," IEEE Transactions on Dielectrics and Electrical Insulation, Vol. 15, no. 3, pp. 678-685, June 2008.

[6] J. K. Nelson and S. Azizi-Ghannad, "Theory and Application of Dynamic Aging for Life Estimation in Machine," IEEE Transactions on Dielectrics and Electrical Insulation, vol. 7, no. 6, pp. 773-782, December 2000.

[7] Tanaka, K. Kojima, H. Onoda, M. Suzuki, K. "Prediction of Residual Breakdown Electrical Field Strength of Epoxy-Mica Paper Insulation Systems for the Stator Winding of Large Generators", IEEE Proceedings of the 19th Electrical Electronics Insulation Conference, pp. 295 299, 1989.

[8] J. E. Timperley and J. R. Michalec, "Estimating the Remaining Service Life Time of Asphaltic-mica Stator Insulation," IEEE Transactions on Energy Conversion, vol. 9, no. 4, pp. 686-693, December 1994.

[9] Hee-dong Kim, Tae-sik Kong, Young-Ho Ju and Byong Han Kim, "Analysis of Insulation Quality in Large Generator Stator Windings", Journal of Electrical Engineering \& Technology Vol. 6, No. 2, pp. 384 390, 2011

[10] Hee-Dong Kim, "Analysis of Insulation Aging Mechanism in Generator Stator Windings", Journal of the KIEEME, Vol. 15, No 2, pp. 119-126, 2002.

\section{Biography}

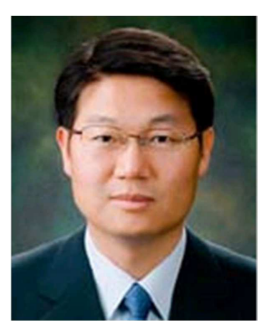

Kyeong-Yeol Kim

$\mathrm{He}$ received his B.S. degree in Electrical Engineering from Chonbuk National University, Jeonju, Korea in 1994 and M.S. degree in Electrical Engineering from Chonbuk National University, Jeonju, Korea in 1996. Since 1997, he has been working for the Korea Electric Power Corporation (KEPCO). He is currently a senior researcher with KEPCO Research Institute, Daejeon, Korea. His research interest is failure analysis of electrical machines in power plant.

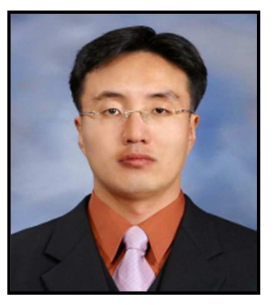

\section{Tae-Sik Kong}

$\mathrm{He}$ received his B.S. degree in Electrical Engineering from Chungbuk National University, Cheongju, Korea in 1997 and M.S. degree in Electrical Engineering from Chungnam National University, Daejeon, Korea in 2004. Since 1997, he has been working for the Korea Electric Power Corporation (KEPCO). He is currently a senior researcher with KEPCO Research Institute, Daejeon, Korea. His research interest is diagnostic test for rotating machine and transformer. 


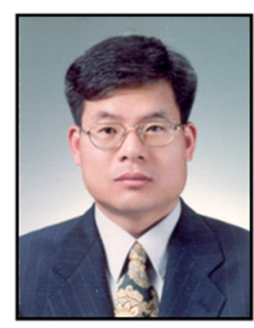

\section{Hee-Dong Kim}

He received the B.S., M.S., and Ph.D. degrees in Electrical Engineering from Hongik University, Seoul, Korea, in 1985, 1987, and 1998, respectively. Since 1990, he has been with Korea Electric Power Corporation (KEPCO) Research Institute, Daejeon, Korea, where he is currently a principal researcher. He was a visiting researcher with the Department of Electrical Engineering, Kyushu Institute of Technology, Kitakyushu, Japan. His research interests include aging mechanisms, diagnostic tests, partial discharge testing, and life assessment for rotating machines, and cable insulation systems.

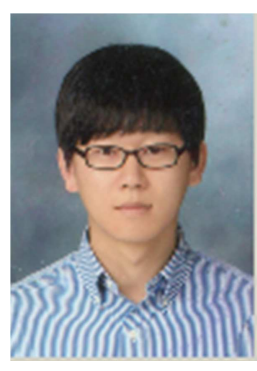

\section{Jae-Heon Park}

He received his B.S. degree in Electrical Engineering from Inha University, Incheon, Korea in 2016. Since 2016, he has been working for the Korea Electric Power Corporation (KEPCO). He is currently a researcher with KEPCO Research Institute, Daejeon, Korea. His research interest is generator model validation and failure analysis of electrical machines.

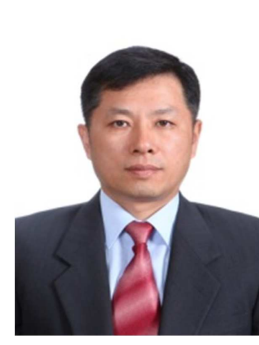

\section{Tae-Sung Park}

He received his B.S. degree in Chemistery from Gongju National University, Gongju, Korea in 2002. Since 1990, he has been working for the Korea Electric Power Corporation (KEPCO). $\mathrm{He}$ is currently a senior researcher with KEPCO Research Institute, Daejeon, Korea. His research interest is diagnostic test for rotating machines. 